\title{
The object-line inferiority effect in pigeons
}

\author{
FRANCISCO J. DONIS and ERIC G. HEINEMANN \\ Brooklyn College, City University of New York, Brooklyn, New York
}

\begin{abstract}
Eight pigeons (Columba livia) were trained to discriminate between diagonal lines presented alone or embedded in a redundant L-shape right-angle form. The stimuli were white and were presented in an environment that was otherwise totally dark. Numerous experiments done with human observers have shown a strong superiority effect when the diagonal lines are embedded in redundant contexts. However, in Experiment 1 of the present study, the pigeons discriminated significantly better between the two diagonal lines when presented alone than when they were embedded in the right-angle context. In order to check on the possibility that these results were restricted to the semi-Ganzfeld condition of Experiment 1, a second experiment was done with black stimuli presented on a white background. Results of Experiment 2 also showed a strong distractor effect. The results of the present experiments confirmed the predictions of the Heinemann and Chase model of pattern recognition by pigeons.
\end{abstract}

A number of experiments done with pigeons have shown that discrimination between two simple visual stimuli is impaired if an identical, and therefore uninformative, element is added to each of these stimuli (Donis, 1992; Heinemann \& Chase, 1990; Steele, 1990). This sort of phenomenon is often referred to as a distractor effect. Heinemann and Chase (1990) have developed a theory of visual pattern recognition by pigeons that offers a quantitative account of this effect. This theory is part of a more general theory of learning, memory, and psychophysical judgment that has been applied, with considerable success, to a variety of phenomena ranging from probability learning to absolute identification of unidimensional and multidimensional stimuli (see Chase, 1983; Chase \& Heinemann, 1989; Heinemann, 1983a, 1983b).

Briefly, the theory assumes that categorization of stimuli is based upon comparison of the visual image induced by current stimulation to each of a small set of stochastically perturbed images retrieved from long-term memory. Each comparison involves a statistical decision process that results in selection of the response that is most likely to produce a favorable outcome. The distractor effect results from the presence of common elements in the patterns presented for discrimination. Such elements add variance to the decision quantity without contributing to the discrimination.

Research on these matters with human observers indicates that the effect of uninformative stimulus elements varies with the forms under study. Under some condi-

Experiment 1 of this study was part of a $\mathrm{PhD}$ thesis submitted by the first author to the City University of New York (Donis, 1992). This research was supported by Grant MH40712 from the National Institute of Mental Health and PSC/CUNY Grant 66911 to the second author. Correspondence concerning this article should be addressed to F. Donis, Department of Psychology, Central Connecticut State University, 1615 Stanley St., New Britain, CT 06050. (e-mail: donis@ccsu.ctstateu.edu).

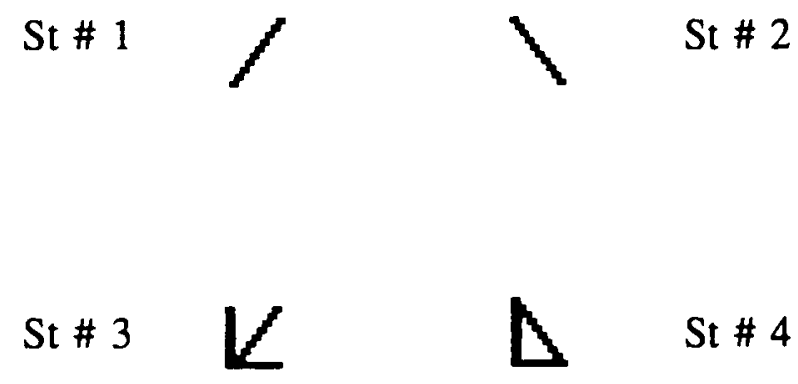

Figure 1. Target lines and target lines in context used in Experiments 1 and 2 .

tions, the addition of uninformative elements yielded distractor effects (e.g., Eriksen \& Eriksen, 1974; Estes, 1972); under others, such elements improved performance (e.g., Enns \& Prinzmetal, 1984; Pomerantz, Sager, \& Stoever, 1977; Weisstein \& Harris, 1974). The latter effect is sometimes referred to as a configural superiority effect. In a particularly strong demonstration of such an effect, Pomerantz et al. (1977) found that choice reaction times for discriminating between two diagonal lines (one with a positive slope and the other with a negative one) were roughly twice as long when these lines were presented alone than when each line was accompanied by an identical visual context (an L-shaped form, see Figure 1). This form of the configural superiority effect has been referred to as the object-line effect (e.g., Enns \& Prinzmetal, 1984; Williams \& Weisstein, 1978).

Though theories have been proposed that deal with either the configural superiority effect (e.g., Chen, 1982; Enns \& Prinzmetal, 1984; Pomerantz, 1981; Pomerantz et al., 1977) or the distractor effect (Estes, 1972, 1974), there appears to be no theory that can predict the result (for human observers) of combining each of two arbitrary patterns with identical contexts of arbitrary form. 
The principal purpose of the present study was to test the Heinemann and Chase theory of pattern recognition by examining how pigeons perform in a stimulus situation that yields a strong object-line effect in human observers. Because the stimulus patterns illustrated in Figure 1 seem to have yielded a particularly robust object-line effect in human observers, we chose to use these patterns in our investigation.

Finding an object-line effect in pigeons would defeat, or at least require major revision of, the Heinemann and Chase theory. On the other hand, finding an object-line inferiority effect predicted by this theory would clearly specify a fundamental difference between pattern recognition by pigeons and humans. This is of particular interest because research on pattern recognition by pigeons has demonstrated remarkable similarities in the way pigeons and humans categorize and identify visual stimuli (Blough, 1984, 1985; Chase \& Heinemann, 1989; Herrnstein, 1984). Though similarities in psychophysical results do not necessarily imply similarities in underlying processes, the idea that the similarities in psychophysical results do reflect similarities in underlying processes is worth considering. If pattern recognition processes were indeed similar in pigeons and humans, then careful examination of systematic differences in these processes could possibly contribute to a better understanding of pattern recognition in both species.

\section{EXPERIMENT 1}

\section{Method}

Subjects. The subjects were four male and four female White Carneaux pigeons (Columba livia; Palmetto Pigeon Plant). Four of the pigeons had participated in previous experiments in which they were trained to discriminate between stimuli that were very different from those used in the present experiment.

The birds were maintained throughout the experiment at $80 \%$ of the mean weight they attained over a 6-week period of free access to food. Between daily experimental sessions, the subjects were housed in a colony room where water and grit were constantly available.

Apparatus and Stimuli. The subjects were trained and tested in a small chamber whose interior was painted flat black (interior dimensions $=51 \mathrm{~cm}$ high, $57 \mathrm{~cm}$ wide, and $46 \mathrm{~cm}$ deep). One wall of this chamber had a large rectangular opening, directly behind which appeared the screen of a Tandy Model CM-11 color monitor. A transparent sheet of Lexan was mounted directly in front of the monitor screen, and in front of this Lexan sheet was a position sensor system based on sets of infrared emitters and detectors (Model 1210; BFANM Corp.). Two small microphones mounted on the Lexan sheet picked up the sounds of the pigeon's pecks on the screen and sent this information to an amplifier. The output of the amplifier combined with the output of the position sensor activated a buzzer, so that the pigeon heard a brief sound whenever it pecked on a region of the screen specified by the experimenter. A small cup into which food pellets (formula $=\mathrm{Cl}, 45 \mathrm{mg}$; P.G. Noyes Co.) were delivered, at appropriate times, by an automatic dispenser was mounted below and to the left of the monitor screen, and a small amber LED was mounted just above the food cup. The LED was turned on whenever a food pellet was delivered and remained on for the duration of the intertrial interval (ITI). All experimental contingencies as well as the form of the stimuli and the recording of data were controlled by a Tandy Model 1000 microcomputer. There were two pairs of stimuli. One pair consisted of two diagonal lines (one with a positive slope and the other with a negative one), to be referred to as targets. Each target was $10 \mathrm{~mm}$ long. The second pair of stimuli consisted of the same two targets, each embedded in a context consisting of two lines that formed an L-shaped pattern (see Figure 1). All stimuli were white presented on a background that appeared totally dark to a moderately darkadapted human observer.

Preliminary training. The subjects were first trained to peck on a large white disk that appeared in the center of the monitor. The size of the disk was gradually reduced until it consisted of four adjacent pixels. Pecks on the disk, of sufficient force to be sensed by the microphone, were followed by reward. In preliminary and discrimination training, reward consisted of two food pellets.

Discrimination training. After completion of preliminary training, each daily training session was divided into 100 discrete trials, separated by 9 -sec ITIs. The screen was uniformly white during the ITI, with a luminance of $75 \mathrm{~cd} / \mathrm{m}^{2}$ as measured by a telephotometer.

At the beginning of each trial, the screen became dark and one of the four patterns shown in Figure 1 appeared in the center of the screen. To ensure that the pigeons viewed the stimuli, they were required to peck at least twice on a $1 \mathrm{~cm} \times 1 \mathrm{~cm}$ region centered on the stimulus pattern. Following these "observing responses," the stimulus pattern disappeared and was replaced by two light blue "response disks," approximately $1.5 \mathrm{~cm}$ in diameter and located to the left and right (with a separation of $6.5 \mathrm{~cm}$ ) and above the location in which the stimulus to be identified had been presented. For 4 of the pigeons, a peck on the left disk was designated the "correct" response to the target that had a positive slope, whether or not the target was accompanied by the L-shaped context. For the remaining 4 pigeons, this relationship was reversed. An incorrect response was followed by a 15-sec ITI in total darkness, and the stimulus incorrectly classified was presented again on the next trial. Performance on repeated trials was excluded from the data analysis.

To accelerate the development of the discrimination, the two stimuli containing the targets of negative slope were presented in white and the ones containing the targets of positive slope were shown in red during the initial phase of discrimination training. This phase of training continued for each subject until its performance met a criterion of $80 \%$ correct or better for 2 successive days. After the criterion was met, the color difference was eliminated (all stimuli were now shown in white). It is only the results obtained after the color cues were removed that represent pure pattern discrimination, of course, and it is only these results that are treated in the analysis that follows.

\section{Results}

Figure 2 shows the learning curves for each of the 8 subjects. Seven of the 8 subjects show better discrimination between the target lines alone than between the same lines in compound with the uninformative visual context. This object-line inferiority effect was remarkably stable over the $\mathbf{5 0}$ daily training sessions. The learning curves of the atypical subject, Bird 5, do not show any systematic difference attributable to the presence or absence of the uninformative visual context.

Because the performance of each of the 8 subjects appears to have reached a reasonably steady level by the last 30 days of training, the results for the last 30 days were combined for a simple statistical analysis. For this purpose, all proportions, $p$, were transformed to arcsine 
- Lines Alone
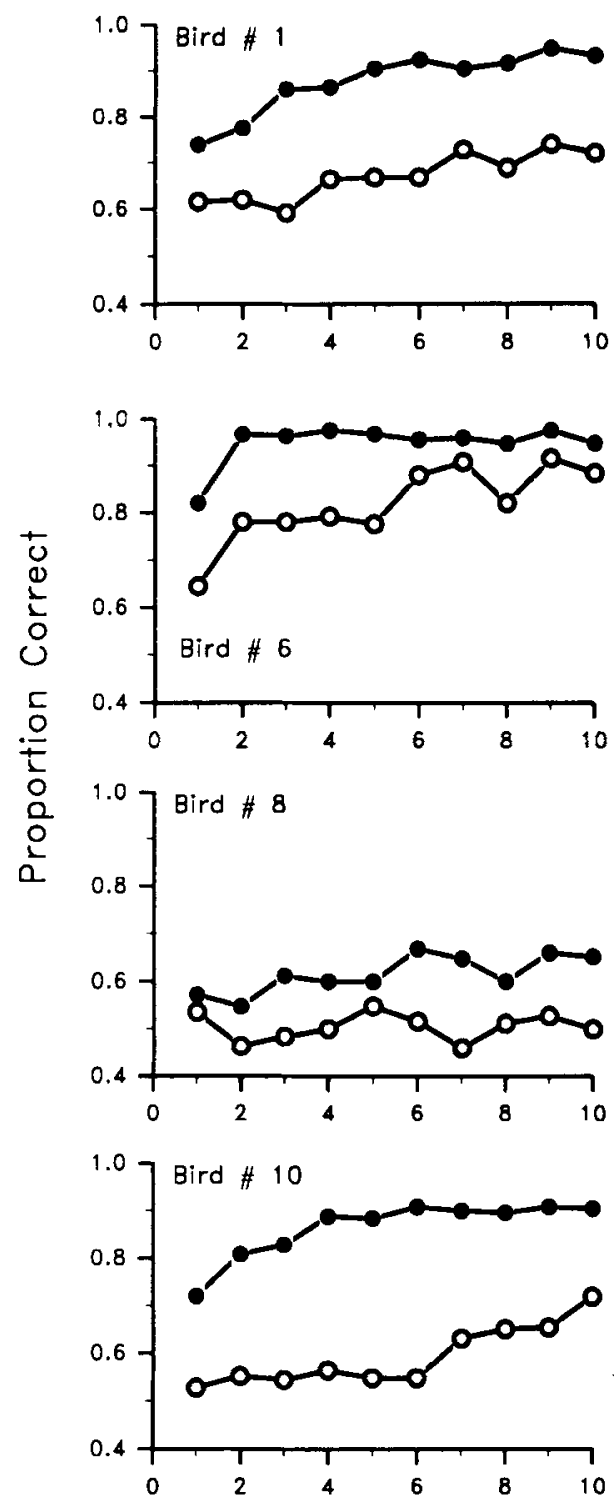

- Lines in Context
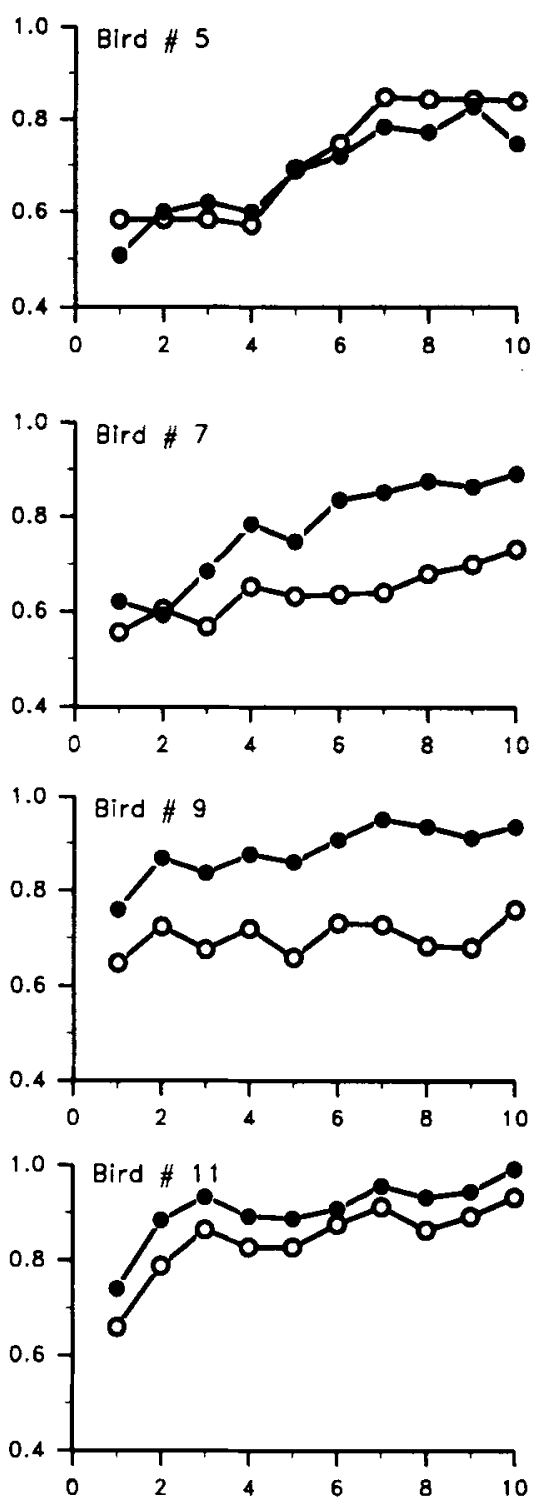

\section{Blocks of 5 Sessions}

Figure 2. Learning curves for each of the 8 subjects of Experiment 1 . Filled circles $=$ lines alone. Open circles $=$ lines in context.

$\left(p^{1 / 2}\right)$. A correlated $t$ test using the transformed proportions showed that discrimination between targets alone was significantly better than discrimination between the targets with the added context $[t(7)=4.05, p<.005]$.

\section{EXPERIMENT 2}

The discriminative stimuli used in Experiment 1 were presented in an environment that contained no visible features or points of reference outside of the patterns studied by the experimenter. Under such circumstances, pigeons seem unable to discriminate between simple visual stimuli that differ only in that one is translated to a different position in the visual field (Donis, 1992; Donis \& Heinemann, 1992). However, pigeons easily learn such position discriminations if the background color of the monitor screen is white, so that the edges of that screen as well as other features of the experimental chamber are visible (Donis, 1992). This finding indicates that, at least under some circumstances, constant features of the en- 


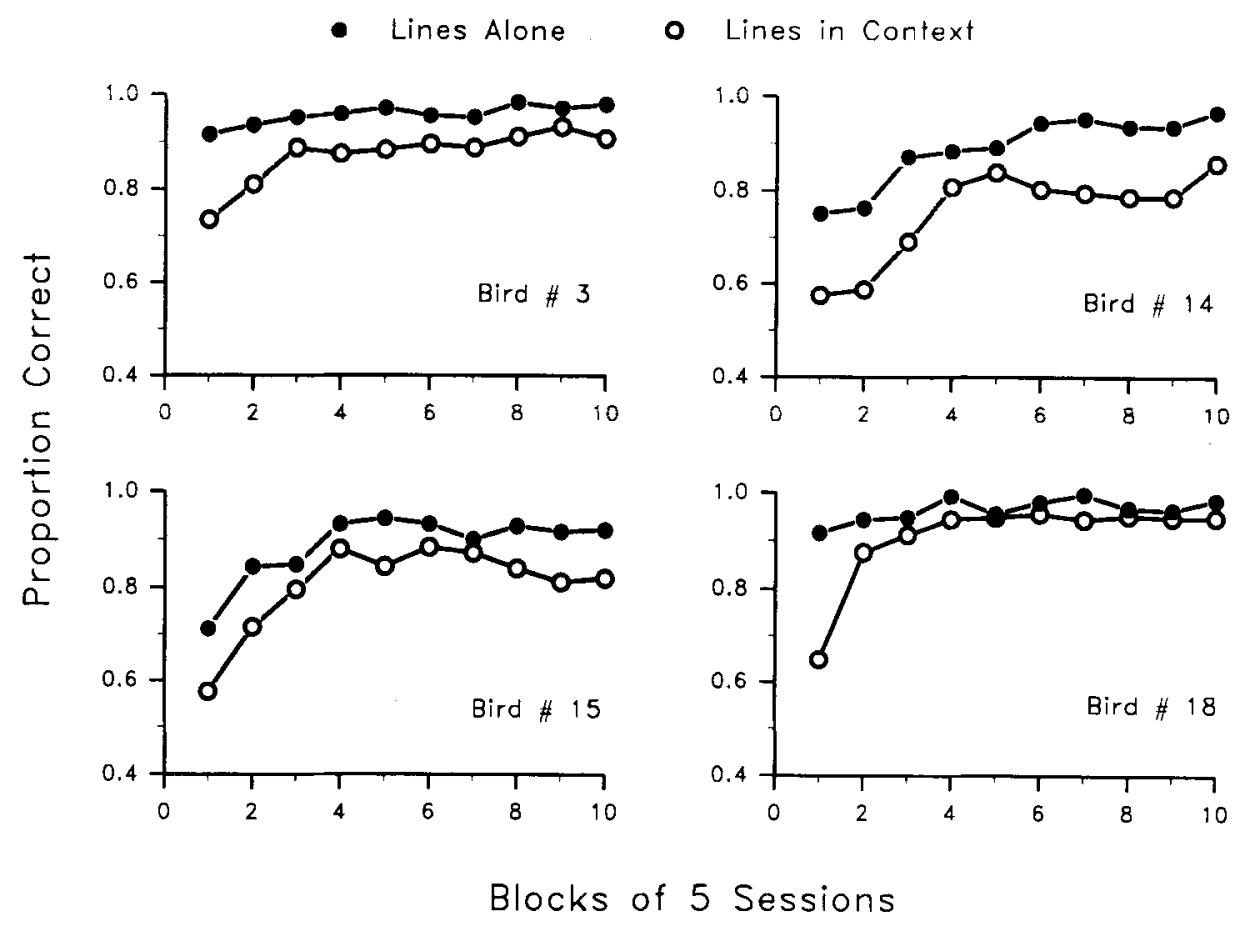

Figure 3. Learning curves for each of the 4 subjects of Experiment 2. Filled circles $=$ lines alone. Open circles $=$ lines in context.

vironment, such as the edges of the screen, constitute part of the pattern of stimulation that is being processed by the birds.

To check on the possibility that the robust object-line inferiority effect found in Experiment 1 might be restricted to the semi-Ganzfeld conditions of that experiment, the sign of the contrast was reversed in Experiment 2.

\section{Method}

Subjects. The subjects were 4 White Carneaux pigeons (Columba livia). All had participated in other experiments that involved stimuli very different from those used in the present one.

Apparatus and Procedure. The apparatus and procedures used were identical to those used in Experiment 1, with the exception that the stimuli were black presented on a white background with a luminance of $75 \mathrm{~cd} / \mathrm{m}^{2}$.

\section{Results}

The learning curves for the 4 subjects of Experiment 2 are shown in Figure 3. The data of each subject show a clear object-line inferiority effect that persisted throughout the 50 sessions of training. The results obtained from the individual subjects during the last $\mathbf{3 0}$ days of training were combined for statistical analysis. The mean proportions correct for the lines-alone and lines-in-context patterns are .9515 and .8777 , respectively.

As in Experiment 1, the results of a correlated $t$ test based on the arcsine-square-root transforms of the proportions showed that discrimination between the targets alone was significantly better than discrimination between the targets in context $[t(3)=4.98, p<.016]$.

\section{DISCUSSION}

The results obtained in Experiments 1 and 2 show effects opposite in direction to those obtained by Pomerantz et al. (1977) with human observers. It is possible that the difference in the results obtained is attributable to the difference in the dependent variables studied: reaction times in the Pomerantz et al. study and proportion correct in the present pigeon experiments. This seems unlikely, however, because there is no reason to expect a speed-accuracy tradeoff in the absence of an incentive for responding quickly. Under these conditions, shorter reaction times are typically associated with higher accuracy scores. It should also be pointed out that in other experiments in which the dependent variable studied was proportion correct (e.g., Enns \& Prinzmetal, 1984; Weisstein \& Harris, 1974), a robust object-line effect was found with human subjects.

Another possibility is that the differences under discussion reflect species differences in early visual processing. Thus, the processes that might be assumed to give rise to "emergent features" in humans (Pomerantz, 1981; Pomerantz et al., 1977) may be absent in pigeons. One difficulty with the view that the configural superiority effects found for human observers reflect early visual pro- 
cessing arises from some results obtained by Enns and Prinzmetal (1984). These investigators found that various contexts that produced superiority effects when presented separately were detrimental to target recognition when the various stimuli were presented within the same experiment. ${ }^{1}$

Another possible explanation for the disparity in the results obtained with pigeons and humans is that human performance in this situation is affected by processes other than visual ones. For example, in experiments that have shown superiority effects, the lines embedded in uninformative contexts resulted in compound patterns that were nameable by humans (e.g., arrows vs. triangles; Pomerantz et al., 1977), but when the lines embedded in uninformative contexts resulted in compound patterns that were unnameable (e.g., diagonal lines embedded in similar uninformative contexts but in a different location; Pomerantz et al., 1977), the result was a distractor effect. It has been shown that, for humans, meaning will enhance discrimination and speed up learning. For example, working with strings of letters, such as IBMPHDCIAFM, Bower and associates (e.g., Bower \& Springston, 1970; Bower \& Winzenz, 1969) found that such strings were learned more accurately if they were presented in "optimal units." These units can be created by inserting spacers, such as blank spaces or zeros (e.g., IBM PHD CIA FM). The resulting meaningful units may then activate their representations in long-term memory, easing the load on shortterm memory (Bower, 1972). No such benefits result when the strings are marked off as nonoptimal units (e.g., IB MPH DCI AFM).

The configural superiority effects obtained by Pomerantz et al. (1977) and Enns and Prinzmetal (1984) might possibly be explained by the meaningfulness of the resulting compound patterns. When a pattern is constructed in an optimal way, so that the compound is more meaningful than its separate components, the contact with its representations in the brain will be facilitated because it may use "dual codes" to decode the information. If the compound is nameable, this pattern may be represented in the linguistic-verbal system and in the pictorial-imagery system. Working with paired associates, Paivio $(1971,1977$, 1978) demonstrated that it is easier to process information that has been encoded in multiple codes. It should be noted, however, that the lines-in-context superiority effect has also been obtained in some situations in which the resulting compound patterns are not readily nameable (e.g., Weisstein \& Harris, 1974).

Since the diagonal lines and the lines embedded in the uninformative elements are arbitrary ones for pigeons, their recognition of these patterns is probably a purely visual process not confounded with verbal or other processes that may be utilized by humans in pattern recognition.

\section{REFERENCES}

BLough, D. S. (1984). Form recognition in pigeons. In H. L. Roitblat, T. G. Bever, \& H. S. Terrace (Eds.), Animal cognition (pp. 277-288). Hillsdale, NJ: Erlbaum.
BLough, D. S. (1985). Discrimination of letters and random dot patterns by pigeons and humans. Joumal of Experimental Psychology: Animal Behavior Processes, 11, 261-280.

BOWER, G. H. (1972). A selective review of organizational factors in memory. In E. Tulving \& W. Donaldson (Eds.). Organizarion of memory (pp. 93-137). New York: Academic Press.

Bower, G. H., \& SPRINGston, F. (1970). Pauses as recoding points in letter series. Joumal of Experimental Psychology, 83, 421-430.

BOWER, G. H., \& WINZENZ, D. (1969). Group structure, coding, and memory for digit series. Joumal of Experimental Psychology Monograph, 80, 1-17

Chase, S. (1983). Pigeons and the magical number seven. In M. L. Commons, R. J. Herrnstein, \& A. R. Wagner (Eds.), Quantitative analyses of behavior: Discrimination processes (Vol. 4, pp. 37-57). Cambridge, MA: Ballinger.

Chase, S., \& HeINEMANN, E. G. (1989). Effects of stimulus complexity on identification and categorization. Intemational Joumal of Comparative Psychology, 3, 165-181.

CHEN, L. (1982). Topological structure in visual perception. Science, 218, 699-700.

Donis, J. F. (1992). The effects of non-informative stimulus elements on pattern recognition by pigeons. University Microfilms Intemational. Dissertation Information Service (Catalog No. 9130312).

Donis, J. F., \& Heineman,, E. G. (1992). Context effects in visual pattern recognition by pigeons. Manuscript in preparation.

EnnS, J. T., \& Prinzmetal, W. (1984). The role of redundancy in the object-line effect. Perception \& Psychophysics, 35, 22-32.

ERIKSEN, B. A., \& ERJKSEN, C. W. (1974). Effects of noise letters upon the identification of a target letter in a nonsearch task. Perception \& Psychophysics, 16, 143-149.

ESTES, W. K. (1972). Interaction of signal and background variables in visual processing. Perception \& Psychophysics, 12, 278-286.

EsTES, W. K. (1974). Redundancy of noise elements and signals in the visual detection of letters. Perception \& Psychophysics, 16, 53-60.

HeinemanN, E. G. (1983a). A memory model for decision processes in pigeons. In M. L. Commons, R. J. Hermstein, \& A. R. Wagner (Eds.), Quantitative analyses of behavior: Discrimination processes (Vol. 4, pp. 3-19). Cambridge, MA: Ballinger.

HeinemanN, E. G. (1983b). The presolution period and the detection of statistical associations. In M. L. Commons, R. J. Hermstein, \& A. R. Wagner (Eds.), Quantitative analyses of behavior: Discrimination processes (Vol. 4, pp. 21-35). Cambridge, MA: Ballinger.

HeinemanN, E. G., \& ChaSe, S. (1990). A quantitative model for pattern recognition. In M. L. Commons, R. J. Hernstein, \& A. R. Wagner (Eds.), Quantitative analyses of behavior: Computational and clinical approaches to pattem recognition and concept formation (Vol. 9, pp. 109-126). Cambridge, MA: Ballinger.

HerRnstein, R. J. (1984). Objects, categories, and discriminative stimuli. In H. L. Roitblat, T. G. Bever, \& H. S. Terrace (Eds.), Animal cognition: Proceedings of the Harry Frank Guggenheim Conference (pp. 233-257). Hillsdale, NJ: Erlbaum.

PAIvio, A. (1971). Imagery and verbal processes. New York: Holt, Rinehart \& Winston.

PArvio, A. (1977). Images, propositions, and knowledge. In J. M. Nichols (Ed.), Images, perception, and knowledge (pp. 47-71). Boston: D. Reidel.

Paivio, A. (1978). A dual coding approach to perception and cognition. In H. L. Pick, Jr. \& E. Saltzman (Eds.), Models of perceiving and processing information (pp. 39-51). Hillsdale, NJ: Erlbaum.

Pomerantz, J. R. (1981). Perceptual organization in information processing. In M. Kubovy \& J. R. Pomerantz (Eds.), Perceptual organization (pp. 141-180). Hillsdale, NJ: Erlbaum.

Pomerantz, J. R., Sager, L. C., \& Stoever, R. J. (1977). Perception of wholes and their component parts: Some configural superiority effects. Joumal of Experimental Psychology: Human Perception \& Performance, 3, 422-435.

Steele, K. M. (1990). Configural processes in pigeon perception. In M. L. Commons, R. J. Hermstein, S. M. Kosslyn, \& D. B. Mumford (Eds.), Quantitative analyses of behavior: Behavioral approaches to pattern recognition and concept formation (Vol. 8, pp. 111-125). Cambridge, MA: Ballinger. 
WeIsstein, N., \& HaRris, C. (1974). Visual detection of line segments An object superiority effect. Science, 186, 752-755.

Williams, A., Weisstein, N. (1978). Line segments are perceived better in a coherent context than alone: An object-line effect in visual perception. Memory \& Cognition, 6, 85-90.

\section{NOTE}

1. Experiments done with human subjects have used a variety of displays and psychophysical methods that differ from ours. However, at least one investigation (e.g., Enns \& Prinzmetal, 1984; Experiment 3) used displays and methods essentially the same as ours but obtained a context superiority effect rather than the distractor effect we obtained in the present experiments.
(Manuscript received January 21, 1992 revision accepted for publication July 2, 1992.) 